\title{
DEGENERAÇÃO LOBAR FRONTOTEMPORAL: POLÊMICAS E DESAFIOS
}

\author{
Ana Paula Pereira Breda ${ }^{1}$, Roberta de Figueiredo Gomes ${ }^{2}$
}

\begin{abstract}
Resumo: As degenerações lobares frontotemporais constituem a segunda causa mais frequente de demência de início precoce, afetando indivíduos que se encontram muitas vezes em idade produtiva. A avaliação neuropsicológica é uma das principais ferramentas para o monitoramento da evolução do processo demencial, sendo um instrumento sensível à identificação de disfunções cognitivas e capaz de direcionar hipóteses diagnósticas. O presente artigo caracteriza-se como uma revisão de literatura não sistemática sobre a temática da degeneração lobar frontotemporal com o foco na avaliação neuropsicológica. Na busca dos artigos utilizou-se o Indexador Medline e a base Portal de Periódicos do CAPES, por consistirem em bases multidisciplinares que reúnem artigos estrangeiros, bem como teses e dissertações brasileiras. Foram selecionados vinte e cinco dentre os cinquenta artigos pesquisados com o foco na área cognitivocomportamental. A pesquisa delimitou o tempo de produção dos artigos nos últimos dez anos utilizando os seguintes termos como palavras-chaves: frontotemporal lobe degeneration, neuropsychological assessment, frontotemporal dementia, degeneração lobar frontotemporal, avaliação neuropsicológica e demência frontotemporal. O artigo revisa a temática da degeneração lobar frontotemporal, abordando brevemente o histórico do conceito e descobertas na área da patologia cerebral, acompanhando a evolução dos critérios diagnósticos e diferenciais das diversas variantes e suas manifestações clínicas. Revisa ainda pesquisas recentes acerca dos perfis cognitivo-comportamentais esperados em cada variante em bateria de avaliação das funções mentais, tecendo considerações sobre a avaliação neuropsicológica nesse contexto. Devese conceber as funções cognitivas como processos conectados em complexas redes neurais, bem como relativizar marcadores frente ao aprofundamento dos conhecimentos na área.
\end{abstract}

Palavras-chave: Degeneração lobar frontotemporal. Avaliação neuropsicológica. Demência frontotemporal.

1 Psicóloga, mestre em psicologia social e da personalidade (PUC-RS). Especialista em teoria e técnica psicanalítica na clínica de crianças, adolescentes e adultos pelo Instituto contemporâneo de psicanálise e transdisciplinaridade. Especialista em Terapia Familiar pelo centro de terapia de casal e família Domus.

2 Psicóloga, Mestre em Medicina e Ciências da Saúde-Neurociências pela Pontifícia Universidade Católica do Rio Grande do Sul, Especialista em Neuropsicologia pela Projecto-Centro Cultural e de Formação, Porto alegre/RS-Brasil. 


\title{
FRONTOTEMPORAL LOBAR DEGENERATION: CONTROVERSIES AND CHALLENGES
}

\begin{abstract}
The Frontotemporal Lobar Degeneration are the second most frequent cause of early-onset dementia, often affecting individuals who are of working age. Neuropsychological assessment is a key tool for monitoring the evolution of the dementia process, a sensitive instrument to detect cognitive dysfunction and able to guide diagnostic hypotheses. The present article is a nonsystematic theoretical review of frontotemporal lobar degeneration on the focus of neuropsychological assessment. The search for articles was made through the Index Medline and the base of CAPES Journal Portal, multidisciplinary bases that gather foreign articles and Brazilian theses and dissertations. It selected twenty-five out of fifty researched articles with a focus on cognitive behavioral aspects. The survey defined the production time of articles in the last ten years using the following terms as keywords: frontotemporal lobe degeneration, neuropsychological assessment, frontotemporal dementia, frontotemporal lobar degeneration, neuropsychological assessment and frontotemporal dementia. It reviews the issue of frontotemporal lobar degeneration, briefly addressing the concept of history and discoveries in the field of brain pathology, following the evolution of diagnostic criteria and the different variants and its clinical manifestations. It also reviews recent research on the cognitive-behavioral profiles expected in each variant assessment battery of mental functions, weaving some thoughts on the neuropsychological assessment in this context. It should grant the cognitive functions as connected processes in complex networks and relativize markers against the deepening of knowledge in the area.
\end{abstract}

Keywords: Frontotemporal lobe degeneration. Neuropsychological assessment. Frontotemporal dementia.

\section{INTRODUÇÃO}

Com o aumento da população idosa no mundo e a preocupação constante com a qualidade de vida durante o envelhecimento, o tema das doenças degenerativas vem adquirindo cada vez mais relevância.

As demências são caracterizadas por déficits que prejudicam o funcionamento ocupacional, laboral e social, representando um declínio de natureza crônica e progressiva no nível de funcionamento do indivíduo (PAULA; FORLENZA, 2012). Dentre as demências pré-senis, a demência frontotemporal (DFT) figura como a segunda causa mais prevalente, respondendo por 5 a 17\% dos casos (RASCOVSKY et al., 2011).

A degeneração lobar frontotemporal abrange síndromes degenerativas heterogêneas que afetam regiões frontais e temporais do cérebro com variações na intensidade e forma de apresentação (BAHIA, 2012).

Ainda que o diagnóstico definitivo das síndromes demenciais dependa do exame anatomopatológico post-mortem, a neuropsicologia tem muito a contribuir no mapeamento de sinais precoces e manifestações das patologias. 
Os avanços da genética identificaram mutações acrescentando muito ao conhecimento das doenças, mas na maioria dos casos essas ainda são desconhecidas, dificultando uma classificação a partir de critérios dessa natureza (CAIXETA, 2010).

A avaliação neuropsicológica pode auxiliar na diferenciação entre o envelhecimento saudável e o patológico, bem como na caracterização das diversas disfunções cognitivas. A análise de padrões de desempenho em testes cognitivos ou de personalidade, aliados a manifestações clínicas, pode sinalizar a presença de alterações, contribuindo para o delineamento de hipóteses diagnósticas.

Esse artigo propõe-se a revisar teoricamente e de forma não sistemática a produção científica dos últimos dez anos na temática da degeneração lobar frontotemporal, sob o enfoque da avaliação neuropsicológica. São abordados aspectos como o histórico, principais síndromes ou variantes, bem como as manifestações cognitivocomportamentais. Finalmente são discutidas questões relacionadas à avaliação neuropsicológica nesse contexto.

\section{METODOLOGIA}

Este artigo constitui uma revisão não sistemática da literatura sob a temática da degeneração lobar frontotemporal. A pesquisa utilizou o Indexador Medline e a base Portal de Periódicos do CAPES, sendo selecionados vinte e cinco dentre cinquenta artigos produzidos na última década, abrangendo o período de 2005 a 2015. Como critérios de exclusão foram desconsiderados artigos que abordassem temas não diretamente relacionados às manifestações clínicas da degeneração lobar frontotemporal ou à avaliação neuropsicológica, sendo excluídos artigos de outras áreas disciplinares como biologia, anatomia e genética.

$\mathrm{Na}$ busca dos artigos utilizou-se o Indexador Medline e a base Portal de Periódicos do CAPES, por consistirem em bases multidisciplinares que reúnem artigos estrangeiros, teses e dissertações brasileiras. Nas bases de dados consultadas foram selecionados apenas dois artigos na língua portuguesa por corresponderam aos critérios estipulados, sendo a maior parte dos artigos produzida fora do Brasil.

A pesquisa delimitou o tempo de produção dos artigos nos últimos dez anos utilizando os seguintes termos como palavras-chaves: degeneração lobar frontotemporal, avaliação neuropsicológica, frontotemporal lobe degeneration and neuropsychological assessment e frontotemporal lobe degeneration, frontotemporal dementia, semantic dementia, progressive aphasia, neuropsychological assessment in frontotemporal degeneration e logopenic aphasia. 


\section{Histórico da degeneração lobar frontotemporal}

A descoberta de que atrofias nas regiões frontais e temporais eram responsáveis por determinados fenótipos e alterações cognitivo-comportamentais surgiu a partir das descrições clínicas de Arnold Pick há mais de um século atrás (BAHIA; TAKADA; NITRINI, 2015; BAHIA, 2012). Pick examinou o cérebro de pacientes com demência e afasia progressiva após a morte, descrevendo atrofias marcantes nos lobos frontais e temporais (PAULOS; MASSANO, 2013). Analisou casos clínicos que cursavam com deterioração cognitiva, afasia progressiva e alterações na conduta social, implicando sintomas comportamentais e de linguagem (PAULA; FORLENZA, 2012; BAHIA; TAKADA; NITRINI, 2015). Posteriormente, Alzheimer apresentou o primeiro estudo sobre alterações microscópicas patológicas, descrevendo os corpúsculos de Pick, emaranhados neurofibrilares e outras inclusões relacionadas a esse tipo de atrofia (BAHIA, 2012). Nessa época, a caracterização das doenças degenerativas se baseava principalmente em achados histológicos post-mortem, não havendo critérios diagnósticos clínicos claros e bem delineados para a sua caracterização.

Em 1994, grupos de pesquisa na Suécia e na Inglaterra de Lund e Manchester publicaram os primeiros critérios clínicos e neuropatológicos para o diagnóstico de atrofias nas regiões frontotemporais, caracterizando a demência frontotemporal (BAHIA, 2012; PAULA; FORLENZA, 2012).

O termo demência frontotemporal (DFT) tornou ultrapassadas designações anteriores como demência do lobo frontal ou do tipo frontal salientando especificamente a síndrome comportamental progressiva. A designação buscou salientar o fato de que esse distúrbio comportamental não estava associado unicamente ao lobo frontal, mas também ao lobo temporal anterior (CAIXETA, 2010).

Os critérios de Lund e Manchester permitiam a caracterização da síndrome a partir da sintomatologia clínica, implicando uma mudança de paradigma em relação ao referencial neuroanatômico. Por outro lado, embora permitissem alguma distinção entre esse tipo de atrofia e patologias como a doença de Alzheimer, careciam de uma definição operacional mais precisa dos sintomas, além de excluir outras patologias associadas aos lobos frontais e temporais (CAIXETA, 2010).

Em 1998, novos critérios foram lançados pelo consenso de Neary, reunindo as várias síndromes associadas à atrofia frontotemporal sob o termo "Degeneração Lobar Frontotemporal (DLFT) ". O conceito buscou contemplar a diversidade de quadros clínicos degenerativos que atingiam essas regiões com características histopatológicas e sintomatológicas diversas (BAHIA, 2012).

As doenças neurodegenerativas são clinicamente caracterizadas pelo declínio cognitivo e funcional e perda gradual de células nervosas em localizações topográficas específicas do sistema nervoso (PAULOS; MASSANO, 2013). 
A DLFT tem sido associada à destruição relativamente seletiva de neurônios nos lobos frontais e temporais, juntamente com preservação de neurônios de regiões posteriores do cérebro (PAULOS; MASSANO, 2013; SHINAGAWA, 2013). Descreve um grupo de patologias caracterizado por atrofia bilateral e frequentemente assimétrica dos lobos frontal e temporal. As manifestações neuropsíquicas que compõe o espectro frontotemporal se correlacionam à distribuição anatômica das lesões que caracterizam o processo neurodegenerativo (PAULA; FORLENZA, 2012).

A DLFT abarca um grupo de distúrbios heterogêneos caracterizados pela atrofia dos lobos temporais e frontais, dentre esses a afasia progressiva primária, a demência semântica e a demência frontotemporal (FELIX-MORAES et al., 2014). Não há consenso entre os autores na classificação dessas três síndromes, sendo que alguns utilizam a designação "variante temporal" para se referir à síndrome semântica e variante comportamental para designar a síndrome comportamental. Há ainda os que consideram a DLFT como componente de um grupo mais amplo denominado Complexo de Pick, incluindo outras síndromes como a degeneração corticobasal e a paralisia supranuclear progressiva.

Neste artigo abordaremos as três principais manifestações associadas à degeneração lobar frontotemporal: a variante comportamental, a afasia progressiva primária (APP) variante agramática/não fluente e APP variante semântica (FELIX-MORAES et al., 2014; BAHIA et al., 2015).

As síndromes apresentam início insidioso e caráter progressivo contando com um quadro clínico característico, com alterações precoces de personalidade, comportamento e de linguagem. Dentre as alterações comportamentais constata-se o isolamento social, a apatia, perda de crítica, desinibição, impulsividade, irritabilidade, inflexibilidade mental e descuido da higiene pessoal. Sintomas depressivos, preocupações somáticas bizarras e estereotipias motoras também podem ocorrer. $\mathrm{Na}$ afasia progressiva, as manifestações incluem a redução da fluência verbal, apraxia, estereotipia e ecolalia.

\section{Variante comportamental da degeneração lobar frontotemporal}

A demência frontotemporal ou variante comportamental constitui uma doença degenerativa heterogênea associada à degeneração lobar frontotemporal ou à variante comportamental da doença de Alzheimer (DA), dependendo de marcadores biológicos e da distribuição da atrofia (CAIXETA, 2010).

Os exames de autopsia tem demonstrado que aproximadamente $20 \%$ dos casos da variante comportamental da DFTL tem patologia consistente com a variante frontal da DA (MCMILLAN et al., 2014). A prevalência da demência frontotemporal tem sido documentada em vários estudos, sendo estimada entre 2.7 e 15.1 dentre 100000 indivíduos com mais de 65 anos de idade. Constitui a terceira demência degenerativa mais comum em países desenvolvidos, após a DA e a dos corpos de Levy, 
com uma incidência de 9.7 a $12 \%$ dentre todas as demências. Os sintomas emergem tipicamente entre os 50 e 60 anos de idade, com uma distribuição entre os gêneros equilibrada, embora as variantes semântica e comportamental costumem aparecer com uma frequência um pouco maior em homens (PAULOS; MASSANO, 2013). Em pessoas com acometimento predominantemente do hemisfério direito, podem ocorrer síndromes como a de Kluver-Bucy, Othelo, Godot, Clerambault e de Diógenes, orientando o diagnóstico para as doenças psiquiátricas (CAIXETA, 2010).

O lobo frontal pode ser dividido em três maiores áreas na direção pósteroanterior; córtex motor, pré-motor e pré-frontal. O córtex pré-frontal comporta três divisões; frontomedial, envolvido principalmente com processos motivacionais; orbitofrontal, que coordena habilidades cognitivas de nível superior no que diz respeito ao comportamento social, atenção e emoções, e o córtex pré-frontal dorsolateral envolvido no controle cognitivo; funções executivas como organização, planejamento, aprendizagem e regulação do comportamento (PAULOS; MASSANO, 2013).

A DFT é identificada nos exames de neuroimagem pela atrofia frontal ou frontotemporal, perda significativa de grandes neurônios corticais frontais, células abalonadas, corpúsculos de inclusão (tipo Pick), degeneração cortical frontal microvascular superficial, ausência de placas senis e novelos neurofibrilares (CAIXETA, 2010).

$\mathrm{Na}$ ausência de biomarcadores definitivos, o diagnóstico de DFT variante comportamental (DFTvc) depende de critérios diagnósticos clínicos para a caracterização das síndromes. Dentre os critérios inicialmente propostos para a DFT figuravam a idade de início anterior a 55 anos, história familiar de distúrbio semelhante, EEG normal, exame neurológico normal, apresentações atípicas de depressão tardia, mania ou psicose e redução do fluxo sanguíneo nas regiões frontais. $\mathrm{Na}$ área comportamental, constam a perda precoce do insight, dos cuidados pessoais e da crítica social e sintomas afetivo-cognitivos. No caso de a patologia vir associada à doença do neurônio motor (DFT-DN), pode ocorrer paralisia bulbar, fraqueza muscular e fasciculações (CAIXETA, 2010).

A definição de critérios pelo consenso de Neary representou um grande avanço para a pesquisa e prática, ainda que conforme alguns autores apresentassem limitações como a ambiguidade dos descritores e a inflexibilidade na sua aplicação. Muitos estudos apontaram a relativa insensibilidade desses critérios nos estágios iniciais da DFTvc, justamente quando as intervenções e tratamentos alcançam maior eficiência (RASCOVSKY et al., 2011).

Em 2011, o Consorcio Internacional de Critérios para a DFTve desenvolveu diretrizes revisadas para o diagnóstico da doença. Apresentou avanços como um menor número de características diagnósticas, nenhuma distinção arbitraria entre características essenciais e secundárias, maior flexibilidade na aplicação, definições operacionais mais claras, inclusão de achados genéticos e neurológicos, critérios 
hierárquicos- distinção entre patologia possível, provável ou definida (RASCOVSKY et al., 2011).

A DFTvc apresenta uma variedade de sintomas que abarcam a áreas diversas do funcionamento psíquico. Na esfera afetiva, são descritos sintomas como a hipocondria, depressão, preocupações somáticas, apatia, falta de espontaneidade, emotividade, embotamento, dificuldades de empatia e modulação dos afetos e perda do insight. Os pacientes podem negar suas deficiências, apresentar mudanças nas suas crenças ou exacerbar traços de personalidade (PAULA; FORLENZA, 2012).

Os sintomas cognitivo-comportamentais incluem hiperoralidade, comportamento perseverativo, compulsivo, estereotipado ou de utilização, desinibição, impulsividade e síndrome de dependência ambiental. Também são relatadas dificuldades no contato social e perda da função comunicativa da linguagem. Em termos gerais, a avaliação neuropsicológica identifica disfunção frontal é circunscrita na ausência de afasia, amnesia ou disfunções perceptivas graves, em um contexto de preservação das praxias e habilidades visuoespaciais (CAIXETA, 2010).

$\mathrm{Na}$ DFTrc, estudos têm constatado que os pacientes conseguem definir o significado das emoções, mas falham em relacionar os estímulos emocionais a cenários descritivos verbais. As dificuldades em identificar os estados emocionais podem contribuir para respostas comportamentais inadequadas (MILLER et al., 2012).

Um estudo recente demonstrou que a magnitude da atrofia em redes ancoradas na amígdala é capaz de predizer a severidade do prejuízo social, enquanto o comprometimento de redes de recompensa do sistema mesolímbico determinam distintos graus de isolamento. (BICKART et al., 2014). Ou seja, pacientes com maior atrofia em redes neurais associadas a dor e à aversão exibem grau importante de falta de apreensão social, enquanto aqueles com atrofia em redes perceptivas demonstram níveis graves de falta de consciência ou compreensão do comportamento social e emocional (BICKART et al., 2014).

\section{Afasia Progressiva Primária}

A afasia progressiva primária (APP) engloba doenças neurodegenerativas que afetam predominantemente o processamento da linguagem, ocorrendo quando o hemisfério dominante é atingido seletivamente pela doença neurodegenerativa (CAIXETA, 2010).

$\mathrm{Na}$ afasia progressiva primária (APP), há deterioração progressiva da linguagem e do discurso durante o período mínimo de dois anos, em um contexto de relativa preservação das demais funções cognitivas (CAIXETA, 2010). O diagnóstico da síndrome é realizado com base no início insidioso e na progressão gradual do prejuízo na linguagem manifesto por déficits no acesso, uso, compreensão das palavras e construção de sentenças. A afasia deve aparecer como o fator principal subjacente ao 
comprometimento das atividades da vida diária, sendo esse aspecto constatado por exclusão a partir da história e exame do paciente (MESULAM et al., 2014). A avaliação da linguagem deve explorar a linguagem - apraxia do discurso, disartria, distorções na voz), a fluência, aspectos gramaticais, a repetição e nomeação e compreensão de palavras e sentenças, a presença de parafasias semânticas ou fonêmicas, o uso e conhecimento dos objetos (MESULAM et al., 2014).

Alterações comportamentais como declínio na conduta social, regulação emocional, embotamento afetivo e perda da capacidade de insight, semelhantes às da apresentação comportamental são encontradas em estágios tardios da APP (FUNAYAMA et al., 2013). Isso reforça a ideia de que as síndromes não são processos estanques, pois no decorrer da evolução do processo degenerativo a atrofia pode se estender a outras áreas antes não afetadas pela doença.

O Consórcio Internacional de 2011 estabeleceu que a APP abarca três variantes principais diferenciadas pelo perfil da afasia e atrofia; a variante semântica, a logopênica e a agramática ou não fluente (GORNO-TEMPINI et al., 2011; CHARLES et al., 2014). As síndromes apresentam características comuns como a proeminência de atrofia assimétrica, perda neuronal, tipo de proteína e hemisfério dominante para a linguagem (MESULAM et al., 2014).

A Afasia Progressiva Primária não fluente (APPnf) é um distúrbio predominantemente da linguagem expressiva, em que problemas na recuperação de palavras ocorrem dentro de um contexto de preservação da compreensão do significado (CAIXETA, 2010). Pacientes com esse tipo de afasia demonstram dificuldades na compreensão de sentenças complexas, não conseguindo relacionar sentenças a figuras correspondentes ou combinar palavras em uma sentença para descrever o que está ocorrendo em determinada ilustração (CHARLES et al., 2014).

A variante não fluente é caracterizada por produção agramática ou apraxia do discurso e por no mínimo duas das três características seguintes: conhecimento de objetos e de palavras singulares preservados e prejuízo na compreensão de sentenças complexas. A disartria também costuma aparecer com alta frequência nessa variante (CROOT et al., 2012).

A existência da variante logopênica como uma entidade distinta ainda é alvo de controvérsias, sendo a definição clínica da síndrome baseada na ausência de déficits que definem as outras variantes da APP. O termo descritivo "logopênica" foi introduzido para designar um tipo de prejuízo da linguagem que parecia peculiar à APP, mas sem que propusesse nenhum critério diagnóstico (LEYTON; HODGES, 2013).

O Consenso Internacional de critérios para o diagnóstico da APP sugeriu que a apraxia do discurso fosse considerada como uma das duas características essenciais da versão agramática da DFTL, enquanto " erros fonológicos na ausência de distúrbios motores da fala" caracterizaria a variante logopênica (CROOT et al., 2012; GORNOTEMPINI et al., 2011). 
A afasia logopênica é definida por prejuízo da recuperação lexical e repetição de sentenças. Diferentemente das outras variantes é comumente associada à doença de Alzheimer, sendo difícil a diferenciação das duas patologias por meio das manifestações clinicas, dependendo para isso de biomarcadores específicos (LEYTON; HODGES, 2013). A variante logopênica com biomarcadores para a DA se manifesta de forma mais agressiva com distúrbios cognitivos e de linguagem mais vastos abrangendo o processamento léxico, semântico e fonêmico, a memória verbal imediata e a praxia ideomotora. Os exames de neuroimagem indicam hipoperfusão mais ampla, envolvendo regiões do lobo parietal (TEICHMANN et al., 2013).

Conforme Rogalski et al. (2007), a prevalência da afasia logopênica em indivíduos com histórico de dificuldades de aprendizagem e dislexia do desenvolvimento é o dobro da encontrada na população normal. Neste tipo de afasia, a disfunção da linguagem não se limita as características típicas como dificuldade em encontrar palavras e memória de trabalho, mas se estende ao sistema linguístico, afetando a produção sintática, a codificação fonológica e as representações semânticas (ROGALSKI et al., 2007).

A variante semântica é uma doença degenerativa caracterizada pela perda seletiva e progressiva do conhecimento conceitual sobre o mundo e à deterioração do conhecimento semântico. Está associada à atrofia assimétrica dos lobos temporais, com predileção específica para o neocórtex anterior, com atrofia variável do hipocampo (TU et al., 2013; LAM et al., 2006; AGOSTA et al., 2009; MANSUR, 2005). Considerada variante da afasia progressiva primária ou direta da DFLT, essa condição tem sido foco de extensas pesquisas há mais de uma década, embora pouco ainda se saiba sobre o perfil neuropsicológico dos pacientes com a síndrome. Isso parece acontecer devido à instabilidade emocional e falta de compreensão e insight que se manifestam desde os estágios iniciais da doença (JEFFERIES; PATTERSON; RALPH, 2006).

O termo "demência semântica" (DS) foi empregado pela primeira vez em 1989, sendo considerada uma forma de demência pré-senil rara, contribuindo para $15 \%$ dos casos de degeneração lobar frontotemporal. A DS é caracterizada por prejuízo significativo na compreensão e nomeação de palavras, bem como no reconhecimento de perceptos visuais, em um contexto de preservação da fluência, gramática, repetição, leitura em voz alta e escrita de palavras regulares (CAIXETA; MANSUR, 2005).

O padrão do comprometimento na DS é caracterizado por uma clara dissociação entre a compreensão de palavras singulares, conhecimento do objeto e déficits na leitura de palavras irregulares, com preservação da fluência, fonologia, sintaxe e memória de trabalho. (AGOSTA et al., 2009). Os sujeitos apresentam redução progressiva da produção verbal e economia de esforço em situação de testagem neuropsicológica, podendo apresentar alterações do comportamento ainda que menos acentuadas que as encontradas na demência frontotemporal (CAIXETA; MANSUR, 2005). O discurso espontâneo é bem articulado, mas caracterizado por anomia e erros semânticos (como usar palavras gerais como "coisa" em vez de nomes específicos). Dicas fonológicas geralmente produzem pouco efeito na precisão da nomeação, enquanto erros 
fonológicos são raros e os pacientes demonstram excelente desempenho na repetição de palavras isoladas ou em tarefas de que envolvam raciocínio fonológico. Em estágios mais avançados, a simples repetição de palavras isoladas pode estar comprometida (JEFFERIES; PATTERSON; RALPH, 2006).

Os estudos de neuroimagem tem comprovado o envolvimento de redes neurais específicas no reconhecimento das emoções vinculado a déficits no processamento de emoções faciais incluindo a amígdala, ínsula e o estriado ventral (MILLER et al., 2012). Em decorrência dessas dificuldades, os pacientes podem apresentar alterações na interação, comportamento social e empatia (MILLER et al., 2012; CAIXETA; MANSUR, 2005).

Como as baterias neuropsicológicas utilizadas na avaliação da memória dependem predominantemente da capacidade de compreensão de material auditivo-verbal e visual, ambas comprometidas na DS, essas podem apontar prejuízo da memória episódica, sendo isso refutado pela preservação da memória do dia-a-dia, característica da doença (CAIXETA; MANSUR, 2005).

\section{DISCUSSÃO}

$\mathrm{Na}$ degeneração lobar frontotemporal, um dos fatores que aumenta a complexidade diagnóstica é a inexistência de uma relação biunívoca entre síndrome e atrofia, não podendo o grau da atrofia ser utilizada como preditor confiável da síndrome clínica que só pode ser determinada pelo exame neuropsicológico (BAHIA et al., 2015; CAIXETA, 2010). Isso indica que não se pode estabelecer uma relação causal entre a gravidade da lesão cerebral e o grau de disfunção cognitiva, já que a síndrome clínica apresenta características que independem do grau da atrofia, como o comprometimento de redes neurais específicas.

A descrição de manifestações clínicas auxilia no delineamento de parâmetros e perfis, contribuindo para o diagnóstico diferencial entre as síndromes, já que a DFT abarca sintomas sociais heterogêneos dependendo do comprometimento dos componentes de determinada rede neural (BICKART et al., 2014).

A análise da produção científica atenta, no entanto, para as limitações da avaliação psicológica quanto à definição diagnóstica, pois ainda que essa possa contribuir na diferenciação entre as diversas síndromes clinicas, se fazem necessários outros marcadores. Além dos biomarcadores, a diferença na distribuição da atrofia constitui outra evidência importante na identificação da patologia subjacente (MCMILLAN et al., 2014; TU et al., 2010). Entrevistas com informantes e a observação longitudinal do funcionamento cognitivo fornecem informações valiosas, aumentando a precisão diagnóstica.

Um dos aspectos debatido em artigos recentes versa sobre o tipo de acometimento da memória em cada variante da DLFT (JEFFERIES; PATTERSON; RALPH, 
2006; AGOSTA et al., 2010). Na variante semântica, as pesquisas têm constatado que os sujeitos podem apresentar dificuldades em diversas tarefas cognitivas secundárias ao prejuízo semântico (JEFFERIES; PATTERSON; RALPH, 2006). Embora os déficits na e memória semântica tenham sido atribuídos à atrofia do lobo temporal anterior, a rede da linguagem constitui um sistema altamente interativo, dependendo de aspectos como a conectividade (AGOSTA et al., 2010). Assim, certas manifestações da síndrome podem estar relacionadas à desconexão entre o lobo temporal anterior e outras áreas da linguagem preservadas. Isso significa que a avaliação da memória verbal, por exemplo, seria mais efetiva se associada à de funções como a linguagem abrangendo aspectos como a fluência verbal, nomeação e diversos outros. Além disso, deve-se considerar que processos complexos como o reconhecimento de faces, o processamento emocional, o raciocínio sequencial e a fluência verbal por dependerem da memória semântica podem estar comprometidos nessa síndrome (JEFFERIES; PATTERSON; RALPH, 2006).

Embora a memória episódica possa estar relativamente preservada (especialmente para material visual), sendo usada como suporte para reaprendizagem do conhecimento sobre palavras e objetos, artigos recentes também têm constatado déficits nessa área (TU et al., 2013; LAM et al., 2006; CAIXETA; MANSUR, 2005). Um estudo de Honolulu identificou sujeitos com queixas subjetivas de memória, déficits na memória episódica e fluência verbal três anos antes do estabelecimento clínico da DS (LAM et al., 2006).

$\mathrm{Na}$ variante comportamental, contrariamente à noção popular de que a memória episódica estaria preservada, pesquisas têm encontrado prejuízo na codificação e consolidação de informações em estágios iniciais da doença (XIE et al., 2010; MATUSZEWSKI et al., 2006). A memória episódica se relaciona à habilidade consciente de codificar, armazenar e recuperar informações sobre eventos previamente vivenciados e envolve tipicamente o resgate de detalhes sobre o evento em um contexto espaço-temporal (IRISH et al., 2014).

Evidências de neuroimagem apontam uma rede neural ampla subjacente a essa função, incluindo os lobos frontais e interações entre regiões pré-frontais e temporais, incluindo estruturas posteriores como o córtex cingulado posterior e parietal lateral. Considerando que as doenças degenerativas estão associadas à deterioração progressiva de regiões cerebrais interconectadas que dão suporte a funções cognitivas complexas, a memória episódica também poderia ser afetada (IRISH et al., 2014).

$\mathrm{Na}$ variante comportamental, o desempenho da memória está relacionado a uma rede neural anterior envolvendo os lobos frontal e temporal anterior. Um crescente corpo de evidências sugere um nível significativo de prejuízo da memória episódica na DFT, compatível com a atrofia no córtex pré-frontal medial e nos lobos temporais ou hipocampo. Estudos com indivíduos saudáveis demonstram que a integridade das regiões mediais e pré-frontais é fundamental para a recuperação de memórias episódicas, concluindo que a presença de graves déficits na memória episódica não deveria afastar o diagnóstico da DLFTvc (IRISH et al., 2014). 
Por outro lado, déficits na memória autobiográfica e/ou episódica podem afetar a experiência consciente da recordação, já que a consciência do self como entidade e experiência subjetiva contínua ao longo do tempo requer a integridade dos processos mnemônicos (SÖDERLUND et al., 2008; MATUSZEWSKI et al., 2006). Indivíduos com doenças neurodegenerativas tendem a superestimar o seu funcionamento em relação à avaliação de informantes, demonstrando pouca crítica acerca de suas dificuldades. A incapacidade de se auto avaliar adequadamente está relacionada à atrofia da região frontal medial direita e do giro temporal medial, envolvendo estruturas específicas (SHANY-UR et al., 2014). A preservação da capacidade demanda habilidades perceptuais, semânticas e mnemônicas, bem como mecanismos atencionais, motivacionais e executivos, sendo possível que o comprometimento de determinados tipos de memória esteja associado à falta de consciência e integridade do self (SHANY-UR et al., 2014). Desta forma, mecanismos nucleares comuns subjacentes aos distúrbios da memória autobiográfica comprometeriam os processos da aprendizagem e armazenamento semântico (MATUSZEWSKI et al., 2006).

Outro tema instigante diz respeito à compreensão gramatical, faculdade que envolve a capacidade de estabelecer relações sintáticas específicas entre as palavras em uma sentença. Os déficits na compreensão gramatical têm sido relacionados à atrofia no lobo frontal esquerdo inferior e temporal anterossuperior (CHARLES et al., 2014). Todos os subgrupos da DFLT apresentam prejuízo significativo na compreensão de sentenças mais complexas, ainda que os mecanismos que intervém nesta função parecem ser diferentes.

$\mathrm{Na}$ variante comportamental, fatores como a memória imediata e as funções executivas interferem na compreensão narrativa, enquanto na versão agramática, os sujeitos não conseguem relacionar sentenças complexas a figuras significativas ou combinar palavras em uma sentença com o contexto de uma ilustração. A dificuldade na compreensão de sentenças complexas parece estar relacionada ao comprometimento da rede léxica na afasia semântica, enquanto na afasia logopênica estaria associada à interrupção de uma rede neural específica (CHARLES et al., 2014).

A revisão teórica dos artigos aponta para uma mudança conceitual importante acerca de uma melhor compreensão das funções mentais no contexto da pesquisa sobre doenças degenerativas. A produção científica recente tem revelado a necessidade de se conceber as funções mentais a partir de uma ótica da complexidade e interconectividade. As pesquisas na área atentam de forma inequívoca para a urgência, no plano da avaliação psicológica, de uma revisão de conceitos e do aprofundamento da compreensão sobre os processos mentais com a finalidade de se descartar percepções errôneas, positivistas e/ou simplistas sobre o funcionamento cognitivo-comportamental. Apontam a necessidade dos profissionais da área da neuropsicologia relativizarem marcadores específicos relacionados a determinadas diretrizes diagnósticas em prol de uma avaliação clínica mais profunda. 


\section{CONCLUSÃO}

Os artigos revisados documentam uma mudança conceitual de uma perspectiva modal para a concepção de processos em redes e conectividade, em que é muito difícil dissociar completamente os elementos. Isso tem implicações importantes para a avaliação neuropsicológica, que tende a conceber as funções cognitivas como módulos que podem ser avaliados singularmente. Isso também ocorre em relação aos conceitos de comportamento e cognição, usualmente pensados como entidades distintas. As pesquisas têm demonstrado que especialmente na área da DFTL esses aspectos se sobrepõem, embora possam aparecer em diferentes intensidades, dependendo do estágio de desenvolvimento da síndrome clínica e do processo degenerativo. Ao mesmo tempo, não se pode perder de vista que estruturas e subdomínios específicos de determinada rede neural podem ser dissociadas de outras redes que conectam determinado tipo de função psíquica.

A maior parte dos artigos revisados neste artigo são provenientes do exterior (via base de dados Medline) visto que o Portal de Periódicos Científicos apontou apenas um artigo relacionado ao tema proposto. Ainda que alguns artigos revelem avanços em relação a uma concepção mais integrada de análise de processos mentais, a maioria tende a investigação empírica de funções singulares, sem estabelecer conexões entre as funções prejudicadas. Por outro lado, demonstram progressos em relação à dissociação de variáveis, contribuindo para a uma maior acurácia diagnóstica.

A avaliação neuropsicológica deve ser capaz de dissociar fatores que possam contribuir para resultados deficitários em bateria de testes, dominando com precisão o tipo de acometimento característico em cada síndrome clínica (CHARLES et al., 2014). No entanto, também deve se propor a relacionar os elementos em um conjunto mais amplo, conectando as diversas funções que participam da realização de uma ou mais tarefas. Questionamentos sobre variantes que podem estar comprometendo determinado desempenho deveriam ser sempre bem-vindos e prontamente acolhidos para que se possa abarcar a complexidade dos fenômenos e evitar interpretações equivocadas.

O conceito "conectividade" auxilia na compreensão do funcionamento de redes neurais responsáveis por determinadas funções psíquicas, veiculando a ideia de um "efeito borboleta", em que habilidades secundárias menos próximas à função atingida e por isso menos imediatamente visíveis possam estar secundariamente comprometidas.

$\mathrm{Na}$ área dos processos demenciais, não se pode perder de vista o papel relevante da avaliação longitudinal, pois a sequência do aparecimento dos sintomas pode diferenciar o tipo predominante de manifestação clínica, sugerindo a patologia subjacente.

$\mathrm{Na}$ temática da DFLT, as pesquisas recentes têm revisto critérios diagnósticos, refutando conceitos que até pouco tempo eram válidos. O comprometimento da memória episódica em fases precoces da variante comportamental, por exemplo, 
constitui um achado que revoluciona critérios diagnósticos diferenciais entre a DFTL e a DA.

A atualização nessa área é indispensável para que se possa contextualizar a avaliação a partir de conhecimentos científicos atuais.

\section{REFERÊNCIAS}

AGOSTA, F. et al. Language networks in semantic dementia. Brain, v.133, n.1, p.286-299, 2009.

BAHIA, V.S. Demência frontotemporal. In E.C. MIOTTO; M.C. SOUZA; M. SCAFF. (org). Neuropsicologia e as interfaces com as neurociências. 2.ed. São Paulo: Casa do Psicólogo, 2012. p.47-54

BAHIA, V.S.; TAKADA, L.T.; NITRINI, R. Demência frontotemporal variante comportamental. In: SANTOS; F.H.; ANDRADE; V.M.; BUENO; O.F.A (Org). Neuropsicologia hoje. Porto Alegre: Artmed, 2015. p. 252-260.

BICKART, K.C. et al. Atrophy in distinct corticolimbic networks in frontotemporal dementia relates to social impairments measured using the Social Impairment Rating Scale. Journal of Neurology, Neurosurgery and Psychiatry, v. 85, n. 4, p. 438-448, 2014.

CAIXETA, L. Demências do tipo não Alzheimer: Demências focais frontotemporais. Porto Alegre: Artmed, 2010.

CAIXETA, L.; MANSUR, L. Demência semântica: avaliação clínica e de neuroimagem. Relato de caso. Arquivos de Neuropsiquiatria, v.63, n.2, p.348-351, 2005.

CHARLES D. et al. Grammatical comprehension deficits in non-fluent/agrammatic primary progressive aphasia. Journal of Neurology, Neurosurgery and Psychiatry, v.85, n.3, p.249256, 2014.

CROOT K. et al. Apraxia of speech and phonological errors in the diagnosis of nonfluent/ agrammatic and logopenic variants of primary progressive aphasia. Journal of Speech, Language and Hearing Research, v.55, n.5, p.1562-1572, 2012.

FELIX-MORAES, R. et al. Frontotemporal dementia: Neuroanatomical correlates of an atypical presentation. BMJ Case Reports. 2014. Disponível em: www. ncbi. nlm. nih.gov/ pmc/articles/articles/MPC4112291/pdf/bcr-2014-205089.pdf. Acesso em: 18 out. 2015.

FUNAYAMA, M et al. Progression of logopenic variant primary progressive aphasia to apraxia and semantic memory deficits. BMC Neurology, v.1, n.13, p.158, 2013.

GORNO-TEMPINI, M.L. et al. Classification of primary progressive aphasia and its variants. Neurology, v.76, n.11, p.1006-1014, 2011.

IRISH, M. et al. Common and unique gray Matter correlates of episodic memory dysfunction in Frontotemporal dementia and Alzheimer's disease. Human Brain Mapping, v.35, n.4, p.1422-1435, 2014. 
JEFFERIES, E.; PATTERSON, K.; RALPH, M.A.L. The natural history of late-stage "pure" semantic dementia. Neurocase, v.12, n.1, p.1-14, 2006.

LAM, L.C. et al. Reduced semantic fluency as an additional screening tool for subjects with questionable dementia. Dementia and Geriatric Cognitive Disorders, v.22, n.2, p.159-164, 2006.

LEYTON, C.E.; HODGES, J.R. Towards a clearer definition of logopenic progressive aphasia. Current Neurology and Neuroscience Reports, v.13, n.11, p.396, 2013.

MATUSZEWSKI, V. et al. Retrieval mechanisms for autobiographical memories: Insights from the frontal variant of frontotemporal dementia. Neuropsychologia, v.44, n.12, p.23862397, 2006.

MCMILLAN, C.T et al. The power of neuroimaging biomarkers for screening frontotemporal dementia. Human Brain Mapping, v.35, n.9, p.4827-4840, 2014.

MESULAM, M.M. et al. Asymmetry and heterogeneity of Alzheimer's and frontotemporal pathology in primary progressive aphasia. Brain, v.137, n.4, p.1176-1192, 2014.

MILLER, L.L. et al. One size does not fit all: Face emotion processing impairments in semantic dementia, behavioural-variant frontotemporal dementia and Alzheimer's disease mediated by distinct cognitive déficits. Behavioural Neurology, v.25, n.1, p.53-60, 2012.

PAULA, V.J.R.; FORLENZA, O.V. Neurobiologia da doença de Alzheimer e outras demências. In: CAIXETA, L. Doença de Alzheimer. Porto Alegre: Artmed, 2012. p.31-47.

PAULOS, J.P.; MASSANO, J. Aspetos clínicos, genéticos e neuropatológicos da demência frontotemporal: atualização e guia. Acta Médica Portuguesa, v.26, n.4, p.392-401, 2013.

RASCOVSKY, K. et al. Sensitivity of revised diagnostic criteria for the behavioural variant of frontotemporal dementia. Brain, v.134, n.9, p. 2456-2477, 2011.

ROGALSKI, E. et al. False recognition of incidentally learned pictures and words in primary progressive aphasia. Neuropsychologia, v.45, n.2, p.368-377, 2007.

SHANY-UR, T. et al. Self-awareness in neurodegenerative disease relies on neural structures mediating reward-driven attention. Brain, v.137, n.8, p.2368-2381, 2014.

SHINAGAWA, S. Phenotypic in the presentation of frontotemporal lobar degeneration. International Review of Psychiatry, v.25, n.2, p.138-144, 2013.

SÖDERLUND H. et al. Episodic memory and regional atrophy in Frontotemporal Lobar Degeneration. Neuropsychologia, v.46, n.1, p.127-136, 2008.

TEICHMANN, M. et al. Deciphering logopenic primary progressive aphasia: a clinical, imaging and biomarker investigation. Brain, v.136, n.11, p.3474-3488, 2013.

TU, S. et al. Dissociation of explicit and implicit long-term memory consolidation in semantic dementia: A case study. Neurocase, v.19, n.4, p.401-407, 2013.

XIE, S.X. et al. Longitudinal patterns of semantic and episodic memory in Frontotemporal Lobar Degeneration and Alzheimer's disease. Journal of the International

Neuropsychological Society, v.16, n.2, p.278-286, 2010. 